\title{
A new multiple Dirichlet series induced by a higher-order form
}

by

Anton Deitmar (Tübingen) and Nikolaos Diamantis (Nottingham)

1. Introduction. This note proposes a new link between two relatively new objects in number theory, higher-order automorphic forms and multiple Dirichlet series.

First-order automorphic forms of weight $k \in 2 \mathbb{Z}_{+}$for a lattice $\Gamma$ in $\mathrm{PSL}_{2}(\mathbb{R})$ are defined as smooth complex-valued functions $f$ on the upper half-plane $\mathfrak{H}$ such that

- $\left.f\right|_{k}(\gamma-1)$ is an automorphic form of weight $k$ for $\Gamma$,

- $\left.f\right|_{k} \pi=f$ for every parabolic element of $\Gamma$,

- $f$ has a "moderate growth at the cusps".

Here the action $\left.\right|_{k}$ of $\mathrm{PSL}_{2}(\mathbb{R})$ on functions $g: \mathfrak{H} \rightarrow \mathbb{C}$ is defined by

$$
\left(\left.g\right|_{k} \gamma\right)(z)=g(\gamma z)(c z+d)^{-k}
$$

with $\gamma=\left(\begin{array}{ll}* & * \\ c & d\end{array}\right)$ in $\mathrm{PSL}_{2}(\mathbb{R})$. We extend this action to the group ring $\mathbb{C}\left[\mathrm{PSL}_{2}(\mathbb{R})\right]$ by linearity. This definition can be extended in a natural way to higher-order forms. (Note that the order in this definition differs from that in the definitions given in previous papers on the subject. The reason for the modification of terminology is related to the fact that, by Fourier transform, $\mathbb{Z}$-supported tempered distributions are mapped to higher-order $\mathbb{Z}$-invariants, where the natural differentiation order of the distributions corresponds to the new notion of order.)

Though some of the ideas behind the investigation of multiple Dirichlet series originated earlier, the systematic study began in the mid-90's ([3], [6], etc.). A definition of multiple Dirichlet series is given in [6]:

$$
\sum_{m_{1}=1}^{\infty} \ldots \sum_{m_{n}=1}^{\infty} \frac{1}{m_{1}^{s_{1}} \ldots m_{n}^{s_{n}}} \int_{0}^{\infty} \ldots \int_{0}^{\infty} \frac{a\left(m_{1}, \ldots, m_{n}, t_{1}, \ldots, t_{l}\right)}{t_{1}^{w_{1}} \ldots t_{l}^{w_{l}}} d t_{1} \ldots d t_{l}
$$

2010 Mathematics Subject Classification: 11F03, 11M32, 11F66.

Key words and phrases: higher-order automorphic forms, $L$-functions, multiple Dirichlet series, Eisenstein series twisted by modular symbols. 
where $a\left(m_{1}, \ldots, m_{n}, t_{1}, \ldots, t_{l}\right)$ is a complex-valued smooth function; more generally, we can take vectors with entries such series. Among these series, those that have a meromorphic continuation to the entire $\mathbb{C}^{n}$ and satisfy enough functional equations are of particular interest for applications and are sometimes referred to as perfect. Constructing perfect multiple Dirichlet series is much harder than the corresponding problem for classical Dirichlet series and it is one of the main aims of the theory. Apart from the multiple Dirichlet series obtained from metaplectic Eisenstein series, essentially none of the known perfect multiple Dirichlet series are constructed as Mellin transforms but are instead obtained by other techniques (cf. [1, 2], [5], etc.). In this note, we construct a perfect multiple Dirichlet series as the Mellin transform of a first-order form. This first-order form is essentially the Eisenstein series twisted by modular symbols. Although the resulting double Dirichlet series has infinitely many poles, and is thus not as suitable for current applications, as it would have been if it had finitely many poles, it is, to our knowledge, the first example of a non-classical automorphic object producing a double Dirichlet series via a Mellin transform. This suggests that there may be a broader class of objects generating perfect multiple Dirichlet series in a systematic way similar to the correspondence between modular forms and Dirichlet series.

2. Eisenstein series twisted by modular symbols. Let $\Gamma \subset \mathrm{PSL}_{2}(\mathbb{R})$ be a non-uniform lattice. As usual we write $x+i y=z \in \mathfrak{H}$. Fix a set $\left\{\mathfrak{a}_{1}, \ldots, \mathfrak{a}_{m}\right\}$ of representatives of the inequivalent cusps of the group $\Gamma$. For each $\mathfrak{a}_{j}$, we consider a scaling matrix $\sigma_{\mathfrak{a}_{j}}$ such that $\sigma_{\mathfrak{a}_{j}}(\infty)=\mathfrak{a}_{j}$ and

$$
\sigma_{\mathfrak{a}_{j}}^{-1} \Gamma_{\mathfrak{a}_{j}} \sigma_{\mathfrak{a}_{j}}=\Gamma_{\infty}=\left\{ \pm\left(\begin{array}{cc}
1 & m \\
0 & 1
\end{array}\right): m \in \mathbb{Z}\right\}
$$

where $\Gamma_{\mathfrak{a}_{j}}$ is the stabilizer of $\mathfrak{a}_{j}$ in $\Gamma$.

Let $\psi: \Gamma \rightarrow \mathbb{C}$ be a group homomorphism which is zero at all parabolic elements. For every $k \in 2 \mathbb{Z}_{+}$and $\mathfrak{a} \in\left\{\mathfrak{a}_{1}, \ldots, \mathfrak{a}_{m}\right\}$, we set

$$
E_{\mathfrak{a}}(z, s, k ; \psi):=\sum_{\gamma \in \Gamma_{\mathfrak{a}} \backslash \Gamma} \psi(\gamma) \operatorname{Im}\left(\sigma_{\mathfrak{a}}^{-1} \gamma z\right)^{s} j\left(\sigma_{\mathfrak{a}}^{-1} \gamma, z\right)^{-k},
$$

where $j(\gamma, z)=c z+d$ when $\gamma=\left(\begin{array}{ll}a & b \\ c & d\end{array}\right)$.

This series is absolutely convergent for $\operatorname{Re}(s)>2-k / 2$ and, for $k=0$, it can be meromorphically continued to all of $\mathbb{C}([\underline{8}])$. It further satisfies

$$
E_{\mathfrak{a}}(\gamma z, s, k ; \psi) j(\gamma, z)^{-k}=E_{\mathfrak{a}}(z, s, k ; \psi)+\psi\left(\gamma^{-1}\right) E_{\mathfrak{a}}(z, s, k)
$$

where

$$
E_{\mathfrak{a}}(z, s, k)=\sum_{\gamma \in \Gamma_{\mathfrak{a}} \backslash \Gamma} \operatorname{Im}\left(\sigma_{\mathfrak{a}}^{-1} \gamma z\right)^{s} j\left(\sigma_{\mathfrak{a}}^{-1} \gamma, z\right)^{-k}
$$

is the classical Eisenstein series at $\mathfrak{a}$. 
If $s$ is not a pole of $E_{\mathfrak{a}}(z, s, k ; \psi)$, then $E_{\mathfrak{a}}(z, s, k ; \psi)$ is a weight $k$ firstorder automorphic form (see [4], where first-order is called second-order according to an older convention) and it has been used to obtain information about the distribution of modular symbols ([7], [10, etc.), the number of appearances of a given generator in reduced words of $\Gamma$ ([8]) etc.

Consider now the lattice

$$
\Gamma^{*}=\left\langle\Gamma_{0}(N), W_{N}\right\rangle=\Gamma_{0}(N) \cup \Gamma_{0}(N) W_{N}
$$

where $\Gamma_{0}(N)=\left\{\left(\begin{array}{ll}a & b \\ c & d\end{array}\right) \in \mathrm{SL}_{2}(\mathbb{Z}): N \mid c\right\}$ and $W_{N}=\left(\begin{array}{cc}0 & -1 / \sqrt{N} \\ \sqrt{N} & 0\end{array}\right)$.

We now concentrate on the case $k=0$, for simplicity and because the weight does not affect the point we want to make. Let $f$ be a newform of weight 2 for $\Gamma_{0}(N)$ such that $L_{f}(1)=0$. We set

$$
\psi(\gamma)=\langle f, \gamma\rangle:=\int_{i \infty}^{\gamma i \infty} f(z) d z
$$

and $E_{\mathfrak{a}}(z, s ; f):=E_{\mathfrak{a}}(z, s ; \psi)$. The Fourier expansion of $E_{\mathfrak{a}}(z, s ; f)$ at $\mathfrak{b}$ is

$$
E_{\mathfrak{a}}\left(\sigma_{\mathfrak{b}} z, s ; f\right)=\phi_{\mathfrak{a} \mathfrak{b}}(s ; f) y^{1-s}+\sum_{n \neq 0} \phi_{\mathfrak{a} \mathfrak{b}}(n, s ; f) W_{s}(n z)
$$

with

$$
\phi_{\mathfrak{a} \mathfrak{b}}(s ; f)=\pi \frac{\Gamma(s-1 / 2)}{\Gamma(s)} \sum_{c \in C_{\mathfrak{a} \mathfrak{b}}} \frac{S_{\mathfrak{a} \mathfrak{b}}(0,0, f ; c)}{c^{2 s}}
$$

and

$$
\phi_{\mathfrak{a} \mathfrak{b}}(n, s ; f)=\frac{\pi^{s}}{\Gamma(s)}|n|^{s-1} \sum_{c \in C_{\mathfrak{a} \mathfrak{b}}} \frac{S_{\mathfrak{a} \mathfrak{b}}(n, 0, f ; c)}{c^{2 s}}
$$

where $C_{\mathfrak{a} \mathfrak{b}}=\left\{c>0:\left(\begin{array}{c}* * \\ c *\end{array}\right) \in \sigma_{\mathfrak{a}}^{-1} \Gamma^{*} \sigma_{\mathfrak{b}}\right\}$ and

$$
S_{\mathfrak{a} \mathfrak{b}}(m, n, f ; c)=\sum_{\substack{\gamma \in \Gamma_{\infty} \backslash \sigma_{\mathfrak{a}}^{-1} \Gamma^{*} \sigma_{\mathfrak{b}} / \Gamma_{\infty} \\ \gamma_{c}=c}}\left\langle f, \sigma_{\mathfrak{a}} \gamma \sigma_{\mathfrak{b}}^{-1}\right\rangle e^{2 \pi i\left(n \gamma_{a} / c+m \gamma_{d} / c\right)} .
$$

We denote the matrix $\left(\phi_{\mathfrak{a} \mathfrak{b}}(s ; f)\right)_{\mathfrak{a}, \mathfrak{b}}$ by $\Phi(s ; f)$.

Set

$$
\mathbf{E}(z, s ; f):=\left(E_{\mathfrak{a}_{1}}(z, s ; f), \ldots, E_{\mathfrak{a}_{m}}(z, s ; f)\right)^{T}
$$

and

$$
\mathbf{E}(z, s):=\left(E_{\mathfrak{a}_{1}}(z, s), \ldots, E_{\mathfrak{a}_{m}}(z, s)\right)^{T}
$$

where ${ }^{T}$ indicates matrix transpose. In [9] it is proved that $\phi_{\mathfrak{a b}}(s ; f)$ and $\mathbf{E}(z, s ; f)$ can be meromorphically continued in $s$ and that

$$
\Phi(s) \mathbf{E}(z, 1-s ; f)=\mathbf{E}(z, s ; f)-\Phi(s ; f) \Phi(1-s) \mathbf{E}(z, s) .
$$


$\Phi(s)=\left(\phi_{\mathfrak{a} \mathfrak{b}}(s)\right)_{\mathfrak{a}, \mathfrak{b}}$ is the scattering matrix of the standard Eisenstein series and satisfies

$$
\Phi(s) \Phi(1-s ; f)=-\Phi(s ; f) \Phi(1-s) .
$$

3. $L$-functions. It is possible to use (3) and (4) to obtain a standard functional equation in $s$ for $\mathbf{E}(z, s ; f)$ (i.e. not "shifted" by a multiple of $\mathbf{E}(z, s))$. Set

$$
\tilde{\mathbf{E}}(z, s ; f):=\mathbf{E}(z, s ; f)-\frac{1}{2} \Phi(s ; f) \Phi(1-s) \mathbf{E}(z, s) .
$$

Then, using the functional equation $\Phi(s) \mathbf{E}(z, 1-s)=\mathbf{E}(z, s)$, we obtain

$$
\begin{aligned}
& \Phi(s) \tilde{\mathbf{E}}(z, 1-s ; f) \\
= & \Phi(s) \mathbf{E}(z, 1-s ; f)-\frac{1}{2} \Phi(s) \Phi(1-s ; f) \Phi(s) \mathbf{E}(z, 1-s) \\
= & \mathbf{E}(z, s ; f)-\Phi(s ; f) \Phi(1-s) \mathbf{E}(z, s)-\frac{1}{2} \Phi(s) \Phi(1-s ; f) \Phi(s) \mathbf{E}(z, 1-s) \\
= & \mathbf{E}(z, s ; f)-\Phi(s ; f) \Phi(1-s) \mathbf{E}(z, s)+\frac{1}{2} \Phi(s ; f) \Phi(1-s) \mathbf{E}(z, s) \\
= & \tilde{\mathbf{E}}(z, s ; f) .
\end{aligned}
$$

In terms of $z,(2)$ and $L_{f}(1)=0$ imply that

$$
\mathbf{E}\left(W_{N} z, s ; f\right)=\mathbf{E}(z, s ; f)+\left\langle f, W_{N}\right\rangle \mathbf{E}(z, s)=\mathbf{E}(z, s ; f)
$$

and thus

$$
\tilde{\mathbf{E}}\left(W_{N} z, s ; f\right)=\tilde{\mathbf{E}}(z, s ; f) .
$$

We next define the "completed" $L$-function of $\tilde{E}_{\mathfrak{a}}$. For every $w$ which is not a pole of $\tilde{E}_{\mathfrak{a}}(i y, w ; f)$ and for $\operatorname{Re}(s)>\max (1+\operatorname{Re}(w), 2-\operatorname{Re}(w))$, set

$$
\tilde{\Lambda}_{\mathfrak{a}}(s, w)=\int_{0}^{\infty}\left(\tilde{E}_{\mathfrak{a}}(i y, w ; f)-a_{\mathfrak{a}}(w) y^{w}-b_{\mathfrak{a}}(w) y^{1-w}\right) y^{s} \frac{d y}{y}
$$

where $a_{\mathfrak{a}}(w) y^{w}+b_{\mathfrak{a}}(w) y^{1-w}$ is the constant term of $\tilde{E}_{\mathfrak{a}}(z, w ; f)$. By the definition of $\tilde{E}_{\mathfrak{a}}(z, w ; f)$, the functions $a_{\mathfrak{a}}(w), b_{\mathfrak{a}}(w)$ are sums of products of entries of $\Phi(w, f)$ and $\Phi(w)$.

We also set

$$
\tilde{\boldsymbol{\Lambda}}:=\left(\tilde{\Lambda}_{\mathfrak{a}_{1}}, \ldots, \tilde{\Lambda}_{\mathfrak{a}_{m}}\right)
$$

We shall prove

TheOREM 3.1. The function $\tilde{\boldsymbol{\Lambda}}(s, w)$ is a (vector-valued) double Dirichlet series which can be meromorphically continued to $\mathbb{C}^{2}$. When $w \notin(1 / 2,1]$, $\tilde{\boldsymbol{\Lambda}}(s, w)$ has simple poles

(i) at the infinitely many poles of $\mathbf{E}(z, \cdot ; f)$ on $\operatorname{Re}(w)=1 / 2$ and

(ii) at $s= \pm w, \pm(w-1)$ if $w$ is not a pole of $\mathbf{E}(z, w ; f)$.

In $(1 / 2,1], \tilde{\boldsymbol{\Lambda}}(s, \cdot)$ has at most finitely many poles. 
$\tilde{\boldsymbol{\Lambda}}(s, w)$ satisfies the functional equations

$$
N^{s} \tilde{\boldsymbol{\Lambda}}(s, w)=\tilde{\boldsymbol{\Lambda}}(-s, w) \quad \text { and } \quad \Phi(w) \tilde{\boldsymbol{\Lambda}}(s, 1-w)=\tilde{\boldsymbol{\Lambda}}(s, w) .
$$

Proof. (6) implies that

$$
\tilde{\Lambda}_{\mathfrak{a}}(s, w)=\int_{1 / \sqrt{N}}^{\infty}\left(\tilde{E}_{\mathfrak{a}}(i y, w ; f)-a_{\mathfrak{a}}(w) y^{w}-b_{\mathfrak{b}}(w) y^{1-w}\right) y^{s} \frac{d y}{y}
$$$$
+\int_{1 / \sqrt{N}}^{\infty}\left(\tilde{E}_{\mathfrak{a}}(i /(N y), w ; f)-a_{\mathfrak{a}}(w)(N y)^{-w}-b_{\mathfrak{b}}(w)(N y)^{w-1}\right)(N y)^{-s} \frac{d y}{y}
$$$$
=\int_{1 / \sqrt{N}}^{\infty}\left(\tilde{E}_{\mathfrak{a}}(i y, w ; f)-a_{\mathfrak{a}}(w) y^{w}-b_{\mathfrak{b}}(w) y^{1-w}\right)\left(y^{s}+N^{-s} y^{-s}\right) \frac{d y}{y}
$$$$
+N^{-s} \int_{1 / \sqrt{N}}^{\infty}\left(a_{\mathfrak{a}}(w) y^{w}+b_{\mathfrak{a}}(w) y^{1-w}-a_{\mathfrak{a}}(w)(N y)^{-w}-b_{\mathfrak{a}}(w)(N y)^{w-1}\right) y^{-s} \frac{d y}{y}
$$$$
=\int_{1 / \sqrt{N}}^{\infty}\left(\tilde{E}_{\mathfrak{a}}(i y, w ; f)-a_{\mathfrak{a}}(w) y^{w}-b_{\mathfrak{b}}(w) y^{1-w}\right)\left(y^{s}+N^{-s} y^{-s}\right) \frac{d y}{y}
$$$$
+\frac{a_{\mathfrak{a}}(w) N^{-\frac{s+w}{2}}}{s-w}+\frac{b_{\mathfrak{a}}(w) N^{\frac{w-s-1}{2}}}{s+w-1}-\frac{a_{\mathfrak{a}}(w) N^{-\frac{s+w}{2}}}{s+w}-\frac{b_{\mathfrak{a}}(w) N^{\frac{w-s-1}{2}}}{s-w+1} .
$$

If $w$ is not a pole of $E_{\mathfrak{a}}(i y, w ; f)$, then $E_{\mathfrak{a}}(i y, w ; f)$ - constant term $=$ $O\left(e^{-\pi y}\right)$ (the implied constant depending on $\left.\left.s\right)(9]\right)$. The analogous fact holds for the entries of $\Phi(w, f) \Phi(1-w) \mathbf{E}(i y, w)$. The last integral of (7) is then well-defined and gives a holomorphic function in $s$. This, together with the meromorphy of $a_{\mathfrak{a}}, b_{\mathfrak{a}}$, shows that $\tilde{\Lambda}_{\mathfrak{a}}$ can be meromorphically continued to $\mathbb{C}^{2}$.

To locate the poles, we first observe that, as shown in [7], $E_{\mathfrak{a}}(z, w ; f)$ has infinitely many simple poles on $\operatorname{Re}(w)=1 / 2$, namely $w=1 / 2+i r_{j}$ with residue proportional to $\eta_{j}(z)$ where $\eta_{j}$ is a Maass form with eigenvalue $1 / 4+r_{j}^{2}$. On the other hand, $\Phi(w)$ and $E_{\mathfrak{a}}(z, w)$ have no poles on $\operatorname{Re}(w)=$ $1 / 2$. This, with Remark 5.5 of [8], further implies that $\Phi(w, f)$ has no poles on $\operatorname{Re}(w)=1 / 2$ either. Therefore, $a_{\mathfrak{a}}(w), b_{\mathfrak{a}}(w)$ have no poles on $\operatorname{Re}(w)=1 / 2$, whereas $\tilde{E}_{\mathfrak{a}}(z, w ; f)$ has infinitely many poles on that line. (7) then implies that $\tilde{\Lambda}_{\mathfrak{a}}$ has infinitely many poles on $\operatorname{Re}(w)=1 / 2$.

The rest of the poles of $E_{\mathfrak{a}}(z, \cdot ; f)$ belong to $(1 / 2,1]$, are simple and they are at most finitely many ([7]). The same fact holds for the functions $\Phi(w, f), \Phi(w), E_{\mathfrak{a}}(z, \cdot)$. A subset of these (which may be proper because of cancellations) will give simple poles of $\tilde{\Lambda}_{\mathfrak{a}}$.

Finally, the above remarks about the location of the poles imply that if a $w \notin(1 / 2,1]$ is not a pole of $\mathbf{E}(z, w ; f)$, it will not be a pole of $\tilde{\mathbf{E}}(z, w ; f)$, 
$a_{\mathfrak{a}}(w)$ and $b_{\mathfrak{a}}(w)$ either. Therefore, by $(7), \tilde{\Lambda}_{\mathfrak{a}}$ will have simple poles at $s= \pm w, \pm(w-1)$.

Regarding the functional equations, (7) first implies that $N^{s} \tilde{\Lambda}_{\mathfrak{a}}(s, w)=$ $\tilde{\Lambda}_{\mathfrak{a}}(-s, w)$.

Further, since the vector of constant terms of the entries of $\tilde{\mathbf{E}}(z, s ; f)$ satisfies the same functional equation in $s$ as $\tilde{\mathbf{E}}(z, s ; f)$ (that is, $(5)$ ), we immediately deduce that $\tilde{\boldsymbol{\Lambda}}(s, w)$ satisfies

$$
\Phi(w) \tilde{\boldsymbol{\Lambda}}(s, 1-w)=\tilde{\boldsymbol{\Lambda}}(s, w) .
$$

Finally, the formulas for the Fourier coefficients of $E_{\mathfrak{a}}(z, s ; f), E_{\mathfrak{a}}(z, s)$, and for the functions $\phi_{\mathfrak{a} \mathfrak{b}}(s), \phi_{\mathfrak{a} \mathfrak{b}}(s, f)$ imply that $\tilde{\Lambda}_{\mathfrak{a}}(s, w)$ is a double Dirichlet series according to the definition in the introduction.

REMARK. The fact that $\tilde{\boldsymbol{\Lambda}}(s, w)$ has infinitely many poles in $w$ shows that $\tilde{\boldsymbol{\Lambda}}$ is a "genuine" double Dirichlet series and not a finite sum of products of the form $L_{f}(s) L_{g}(w)$, with the sum ranging over pairs of classical modular forms $(f, g)$. Indeed, for each fixed $s$, such a sum can only have at most finitely many poles in $w$, because the $L$-function of a modular form has at most two poles.

Acknowledgments. The authors thank Dorian Goldfeld for many useful conversations during work on this note and the referee for a careful reading of the manuscript.

\section{References}

[1] B. Brubaker, D. Bump, G. Chinta, S. Friedberg and J. Hoffstein, Weyl group multiple Dirichlet series I, in: Multiple Dirichlet Series, Automorphic Forms, and Analytic Number Theory, Proc. Sympos. Pure Math. 75, Amer. Math. Soc., 2006, 91-114.

[2] B. Brubaker, D. Bump, S. Friedberg and J. Hoffstein, Weyl group multiple Dirichlet series III: Eisenstein series and twisted unstable $A_{r}$, Ann. of Math. 166 (2007), 293-316.

[3] D. Bump, S. Friedberg and J. Hoffstein, On some applications of automorphic forms to number theory, Bull. Amer. Math. Soc. 33 (1996), 157-175.

[4] G. Chinta, N. Diamantis and C. O'Sullivan, Second order modular forms, Acta Arith. 103 (2002), 209-223.

[5] A. Diaconu and D. Goldfeld, Second moments of quadratic Hecke L-series and multiple Dirichlet series I, in: Multiple Dirichlet Series, Automorphic Forms, and Analytic Number Theory, Proc. Sympos. Pure Math. 75, Amer. Math. Soc., 2006, 59-89.

[6] A. Diaconu, D. Goldfeld and J. Hoffstein, Multiple Dirichlet series and moments of zeta and L-functions, Compos. Math. 139 (2003), 297-360.

[7] D. Goldfeld, The distribution of modular symbols, in: Number Theory in Progress, in honor of A. Schinzel (Zakopane, 1997), Vol. 2, de Gruyter, 1999, 849-865.

[8] D. Goldfeld and C. O'Sullivan, Estimating additive character sums for Fuchsian groups, Ramanujan J. 7 (2003), 241-267.

[9] C. O'Sullivan, Properties of Eisenstein series formed with modular symbols, J. Reine Angew. Math. 518 (2000), 163-186. 
[10] Y. Petridis and M. S. Risager, Modular symbols have a normal distribution, Geom. Funct. Anal. 14 (2004), 1013-1043.

Anton Deitmar

Mathematisches Institut

Auf der Morgenstelle 10

72076 Tübingen, Germany

E-mail: deitmar@uni-tuebingen.de
Nikolaos Diamantis School of Mathematical Sciences

University of Nottingham

University Park

Nottingham NG7 2RD, United Kingdom E-mail: nikolaos.diamantis@nottingham.ac.uk

Received on 3.11.2008

and in revised form on 12.1.2009 